\title{
Self-contained actuation of phase-change pistons in microchannels
}

\author{
Rohit Pal ${ }^{1}$ and Mark A Burns ${ }^{1,2}$ \\ ${ }^{1}$ Department of Chemical Engineering, University of Michigan, Ann Arbor, MI 48109, USA \\ ${ }^{2}$ Department of Biomedical Engineering, University of Michigan, Ann Arbor, MI 48109, \\ USA \\ E-mail: maburns@umich.edu
}

Received 25 October 2005, in final form 13 February 2006

Published 14 March 2006

Online at stacks.iop.org/JMM/16/786

\begin{abstract}
An inchworm-like self-contained actuation mechanism for phase-change pistons using a movable thermopneumatic air chamber has been demonstrated. Two phase-change pistons that enclose an air pocket between them have been moved incremental distances by successive melting and solidification of the pistons timed with the expansion and contraction of the air pocket. For a system in which both pistons were wax-based, localized heating of each piston and the air chamber was used to achieve the motion of the assembly. This design used a two-component wax piston that did not coat the microchannel walls. In a separate design a combination of a wax piston with a piston that solidifies at high temperatures was used to eliminate the localized heating requirement for self-contained actuation. This actuation mechanism can be easily integrated on lab-on-a-chip devices for the self-contained pumping of fluid columns.
\end{abstract}

\section{Introduction}

One of the main challenges in designing microfluidic control elements is the conformability of the component with the microchannel cross-section. For macro fluidic systems the flow control components can be precisely machined to match the flow conduit within acceptable tolerances. However in the microfluidic systems not only is this hard to fabricate, the error tolerances are several orders of magnitude more stringent owing to overall reduction in size. Several clever designs that use membranes and cantilevers to mimic macro-systems have been fabricated to minimize this problem [1].

More recently novel microfluidic components designed especially for the micro-scale have been developed, the most prominent of which are based on soft lithography and conformable pistons. The soft lithographic approach uses a flexible polymer for device construction, and flow control is achieved by pinching off the required channels [2]. This control has been done internally using an overlapping layer of control channels and externally using pneumatic [3] or mechanical $[4,5]$ actuation. Conformable pistons that can deform to seal the cross-section of microchannels in devices made from more rigid substrates have been implemented successfully. Pistons made of a variety of materials including polymers [6-8], ferrofluids [9, 10] and electrorheological fluids [11] have been used depending on process requirements and complexity afforded.

Pistons made of materials whose phase can be reversibly changed from solid to liquid offer added advantages for microfluidic control. The underlying principle for the actuation of these pistons is that in the liquid state the viscosity of the piston is low making it mobile while in the solid state the piston is immobile and forms a strong hermetic seal. The most common mode for inducing phase change has been the thermal mode. The simplest demonstration of this was the freezethaw valve where the process fluid was solidified in the desired locations by lowering the temperature locally [12]. In contrast, thermoreversible polymers that solidify when the temperature is raised have also been used for valving applications [13, 14]. However using a piston that is solid at ambient conditions is advantageous because no energy is required to maintain the stable immobile state thus allowing the piston or valve to be latched.

Pneumatically and thermopneumatically actuated phasechange pistons have been successfully integrated on microdevices $[15,16]$. These include valves that use a waxbased meltable piston actuated pneumatically by pressure and vacuum to close and open a fluidic channel [17]. The motion 
of the molten piston was controlled by adjusting the time and intensity of a pneumatic pressure pulse. Another mechanism for controlling the position of the plug involved spatial thermal gradients along the microchannel such that the advancing edge of the piston solidified at the desired location when it encountered a temperature region below its melting point. Self-contained thermopneumatic actuation using on-chip air chambers to operate single-use paraffin valves has also been demonstrated [18]. However current designs of air chambers for thermopneumatic actuation require large air chambers and high temperatures for moving even moderate distances.

In this work a self-contained thermopneumatic actuation principle for phase-change pistons using a moving air chamber has been presented. The system consists of two phase-change pistons with an air pocket between them. Selective melting of the pistons coupled with heating of the enclosed air chamber has been used for incremental inchworm-like motion of the assembly. Individual pistons have been designed so that they move without coating the walls of the channels. Two different designs for the inchworm thermopneumatic actuation have been presented. The first design uses two wax-based pistons and requires localized heating while the second design consists of a wax and a thermoreversible piston that can be actuated by global heating of the microdevice.

\section{Materials and methods}

\section{Device fabrication and assembly}

Heating elements consisting of $300 \AA$ titanium and $1000 \AA$ platinum were evaporated on a silicon wafer $(\langle 100\rangle, 500 \mu \mathrm{m}$ thick, $10 \mathrm{~cm}$ diameter) that was patterned using standard photo-lithography described earlier [17]. After performing a liftoff procedure using acetone (CMOS grade; J T Baker, Phillipsburg, NJ, USA) the wafer was diced and the individual devices were fixed on a custom-made printed circuit board using a steel weld epoxy (ITW Performance Polymers, Riviera Beach, FL). 2 mil gold wirebonds were used to make electrical connection between the device and the printed circuit board (Kulicke Soffa 4124 ball bonder). A $5 \mu \mathrm{m}$ parylene film was deposited on the device (PDS 2010 LABCOTER $^{\circledR} 2$; Specialty Coating Systems, Indianapolis, IN). The wirebonds were encapsulated using an electric grade epoxy (EP939, Thermoset, Indianapolis, IN).

Microchannels were etched in photo-lithographically patterned borofloat glass wafers $(500 \mu \mathrm{m}$ thick, $10 \mathrm{~cm}$ diameter) using hydrofluoric acid $(49 \% \mathrm{HF}, \mathrm{CMOS}$ grade; $\mathrm{J} \mathrm{T}$ Baker). The wafer was thermally annealed $\left(560{ }^{\circ} \mathrm{C}\right.$ for $1 \mathrm{~h})$ prior to patterning and thin films of chromium (600 $\AA$ ) and gold (4000 $\AA$ ) along with hard-baked photoresist were used at the etch mask. The glass wafers were diced to yield individual devices. An electrochemical spark discharge apparatus was used to drill access holes ( $~ 300 \mu$ m diameter) in the glass devices. The glass device was bonded to the silicon substrate using a UV-cured optical adhesive (NOA 72; Norland Products, Cranbury, NJ) that was cured in UV light $(365 \mathrm{~nm})$ for $6 \mathrm{~h}$ and then in an oven at $50{ }^{\circ} \mathrm{C}$ for $12 \mathrm{~h}$.

\section{On-chip heater control}

For measuring temperature on the microdevice the on-chip resistance temperature detectors (RTDs) were calibrated. This was done by heating the entire microdevice in an oven and using a MIO-PCI6031E board to output the temperatureresistance data for the RTDs to a computer. This calibration curve was recorded by a LABVIEW (National Instruments) VI. For controlling the temperature $\left( \pm 1^{\circ} \mathrm{C}\right)$ another Labview VI regulated the voltage across the heaters employing a feedback proportional-integral control using the AO32PCI6704 board. The heaters were connected to the power supply through a signal conditioning circuit to boost the supply voltage from the computer. For thermal isolation on the microdevice the PCB was routed under the silicon and the cavity was refilled with a high-conductivity siliconebased thermal grease (product no. 54013, AOS Thermal Compounds, Eatontown, NJ). The entire device was placed on a temperature-controlled chuck or a Peltier device. Energy pulses given to the on-chip heaters were also regulated using LABVIEW ER-16 relay boards interfaced with the computer through the DIO96 board.

\section{Results and discussion}

\section{Basic concept}

Self-contained motion of phase-change pistons in microchannels can be achieved by using an assembly of two pistons separated by an air chamber (figure 1(a)). Thermopneumatic motion of this assembly was obtained by melting the first piston and heating the air pocket while the second piston is maintained in an immobile state (figure 1(b)). As shown in figure 1(c), heating the air between the pistons propels the first piston forward. The first piston is then solidified in the advanced position (figure $1(d)$ ). The second piston is then made mobile, and cooling the air between the pistons creates a vacuum that pulls the second mobile piston forward (figures $1(e)$ and $(f)$ ). Finally both pistons are solidified in the new position (figure $1(g)$ ). Repeating this cycle moves the assembly forward in small steps resulting in an inchworm-like motion. This piston assembly that hermetically seals the microchannel crosssection can be used to push or pull liquid columns located either in front of the leading piston or behind the trailing piston.

The first step for realizing the self-contained actuation is the assembly of the two wax pistons separated by an air gap. A single plug of a combination wax (75\% M1595 and 25\% Lycojet wax) was first loaded through an inlet hole and pumped pneumatically into the microchannel. For loading the second piston, wax was again placed at the inlet hole and melted such that it seals the entire inlet hole. The second plug was then pulled into the microchannel by the self-contained actuation procedure mentioned in the last section. Though this serial loading process was used here, a microfabrication technique to pattern the phase-change pistons would be more practical for actual applications [19].

Self-contained motion was demonstrated by using two pistons of a combination wax that was loaded as described in the previous paragraph. These plugs were loaded into a $300 \mu \mathrm{m}$ wide $\times 50 \mu \mathrm{m}$ deep microchannel and had a $1 \mathrm{~mm}$ long air pocket between them at room temperature (figure 2(a)). The actuation process began by melting the 


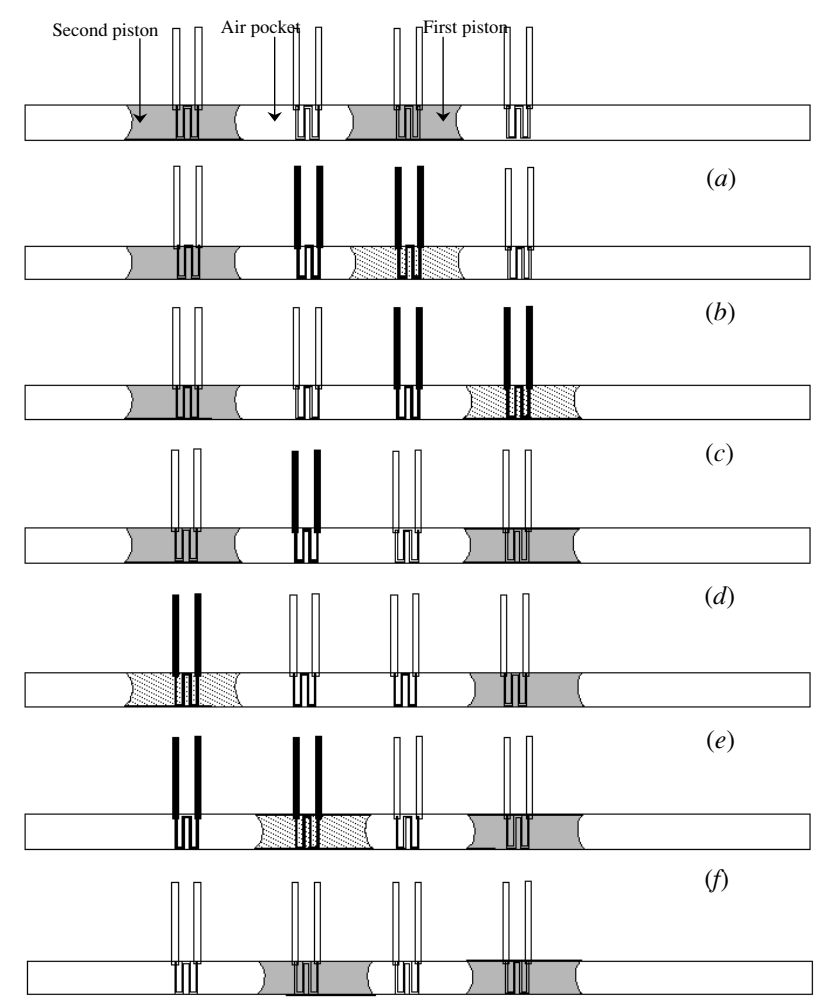

$(g)$

Figure 1. Operation schematics of an inchworm thermopneumatic pump using two wax pistons. (a) Assembly of two pistons separated by an air chamber. (b) The first piston is melted and the enclosed air pocket is heated. (c) The first piston is propelled forward in the molten state. $(d)$ The first piston is solidified in the advanced position. (e) The second piston is melted. $(f)$ Cooling the air between the pistons creates a vacuum that pulls the second piston forward. $(g)$ Both pistons are solidified in the new position. Only the heaters in solid black are actuated for each step. first piston $\left(90^{\circ} \mathrm{C}\right)$ and heating the air pocket to propel the molten piston forward (figure $2(b)$ ). The second piston was solid in this step and did not move. Next, the heaters below the first piston and the air pocket were turned off. This solidified the first piston and created a vacuum between the pistons. At this point the heaters below the second piston were actuated and the molten piston was pulled forward (figure 2(c)). All heaters were then turned off to solidify the pistons in the new position (figure $2(d)$ ). Since the configuration is symmetric the assembly can be moved in either direction.

Though this mechanism can be used to achieve pumping, temperature localization is required for synchronized phase change of pistons and heating/cooling of the air chamber. In the current system, temperature localization was achieved by keeping the device in contact with a surface at $5{ }^{\circ} \mathrm{C}$ that prevented the lateral spread of heat. For better performance of the system advanced thermal isolation schemes using a suspended heater design can be incorporated [20]. These schemes can give temperature gradients of $\sim 160{ }^{\circ} \mathrm{C} \mathrm{m}^{-1}$ as compared to $\sim 50{ }^{\circ} \mathrm{C} \mathrm{m}^{-1}$ achieved by the simple heat sink scheme.

\section{Stabilized wax pistons}

The motion of the wax pistons is primarily governed by the rheology of the phase-change material. For most singlecomponent waxes the melting temperature is well defined and complete phase transition occurs within a few degrees (figure 3). Single-component waxes exhibit no motion in the solid state (i.e. below their melting point), and in the molten state their motion can be quantified by the Hagen-Poiseuille equation [21]. Though these systems are easy to design, the pistons are susceptible to 'punch through', a phenomenon well studied in the gas-induced-injection-molding literature [22]. As shown in figure 4, punch through occurs due to the

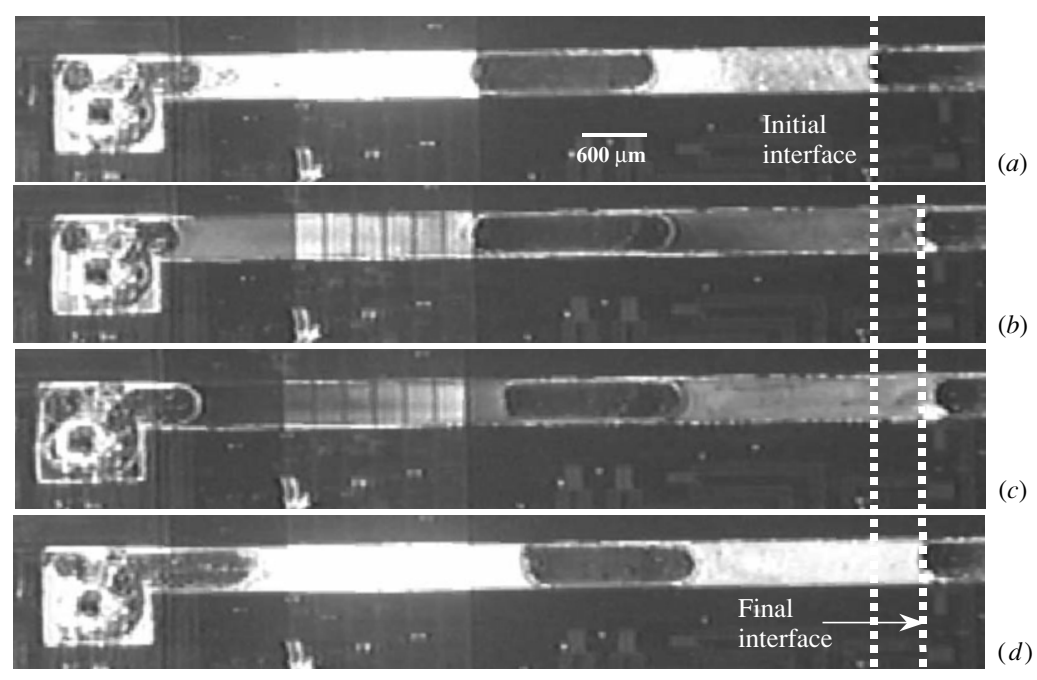

Figure 2. Operation pictures of an inchworm thermopneumatic pump using two wax pistons in a microchannel $300 \mu \mathrm{m}$ wide $\times$ $50 \mu \mathrm{m}$ deep. (a) The initial position of the pistons. (b) The first piston is melted and the air pocket is heated to propel the piston forward. (c) The first piston is solidified and the second piston is melted. The air pocket is cooled down to pull the second piston forward. (d) Both pistons are solidified. 


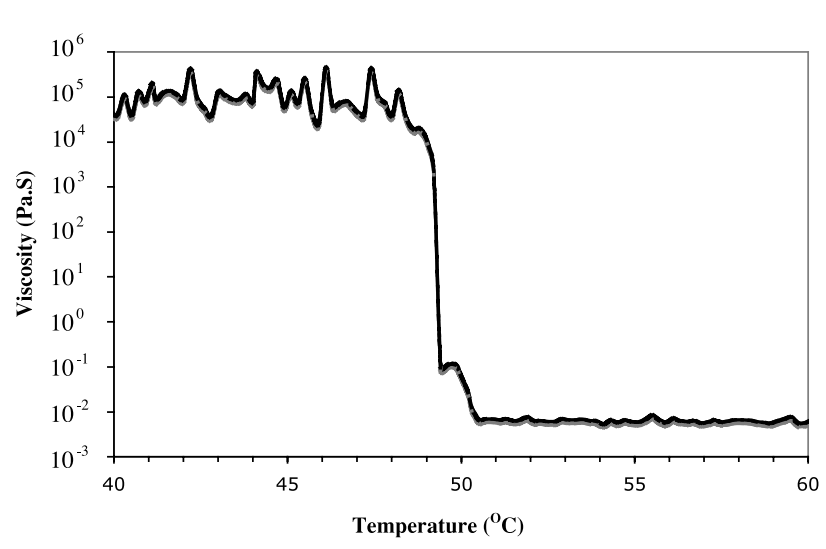

Figure 3. A typical plot of viscosity as a function of temperature for a single-component wax. The wax used in this experiment was a paraffin wax that melted around $50{ }^{\circ} \mathrm{C}$. The measurements were made on a constant stress rheometer using the oscillatory mode at a frequency of $1 \mathrm{~Hz}$.

instability of the interface and the coating of the microchannel walls. Continuous loss of material as the piston moves forward eventually compromises the integrity of the piston and the air column punches through the middle.

Two-component wax mixtures exhibit a complex rheology that can be used to achieve stable piston motion and prevent punch through. Figure 5 shows the viscosity as a function of shear rate at different temperatures for one such mixture: $75 \%$ M1595 and 25\% Lycojet (by weight). The M1595 wax melts in the range of $83-85^{\circ} \mathrm{C}$ while the Lycojet wax melts around $50{ }^{\circ} \mathrm{C}$. As seen from the figure, in the temperature range above the melting point of the M1595 wax (region 1) this combination wax is a low viscosity melt that is almost Newtonian. Below the melting point of the Lycojet wax (region 2) the combination wax is a solid. However in the temperature range between the melting points of the M1595 and the Lycojet waxes (region 3), the behavior is much different. The viscosity remains high up to a certain shear rate after which it suddenly drops by several orders of magnitude. This yielding or 'slip' may be due to the presence of the Lycojet wax that is in the molten state.

Stable motion of a two-component wax piston in a microchannel is shown in figure 6. This stable motion is achieved because the wax mixture slips at the wall instead of spreading over; the shear stress is the highest at the wall and hence the piston yields first at the wall. The stress-induced slip-like behavior occurs in the temperature range between the melting points of the two waxes. The integrity of the plug is further improved due to the fact that one of the components is in the solid phase. This flow profile prevents continuous deposition of material on the wall.

The 'slip' phenomenon in the microchannel was verified by measuring the wall shear stress when the piston yielded as a function of temperature and correlating it to the values obtained using the rheometer. The wall shear stress is given by

$$
\tau_{\text {wall }}=0.5 d(\Delta P / L)
$$

where $d$ is the channel depth, $\Delta P$ is the pressure difference between the two sides of the plug and $L$ is the length of the plug. Figure 7 shows the yield stress as a function of temperature as measured by moving molten drops in a microchannel. As seen from the figure the values of the yield stress are in close agreement with that measured using the rheometer.
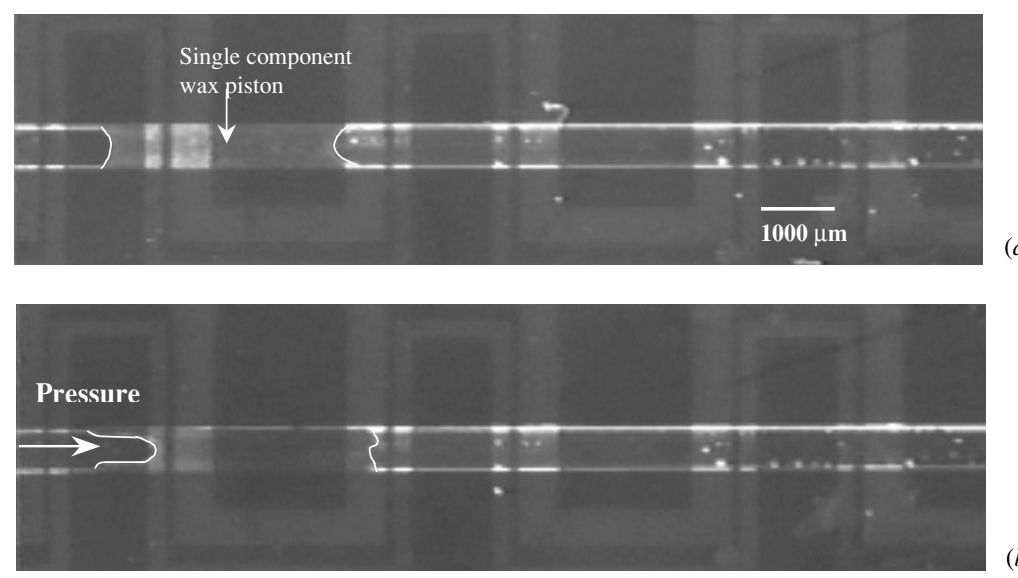

(b)

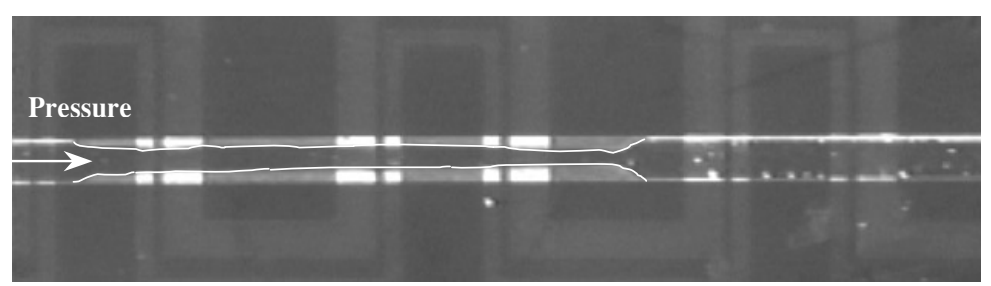

$(c)$

Figure 4. Pictures of a phase-change piston that was punched through by air. (a) The initial plug. (b) Wax coating on the wall can be seen as the piston moves in the microchannel. $(c)$ Punch through of the piston when the integrity of the plug is compromised by the air column. The piston was about $3 \mathrm{~mm}$ long and a pressure of about $0.5 \mathrm{psi}(\sim 3450 \mathrm{~Pa})$ was applied for the motion of the piston. 


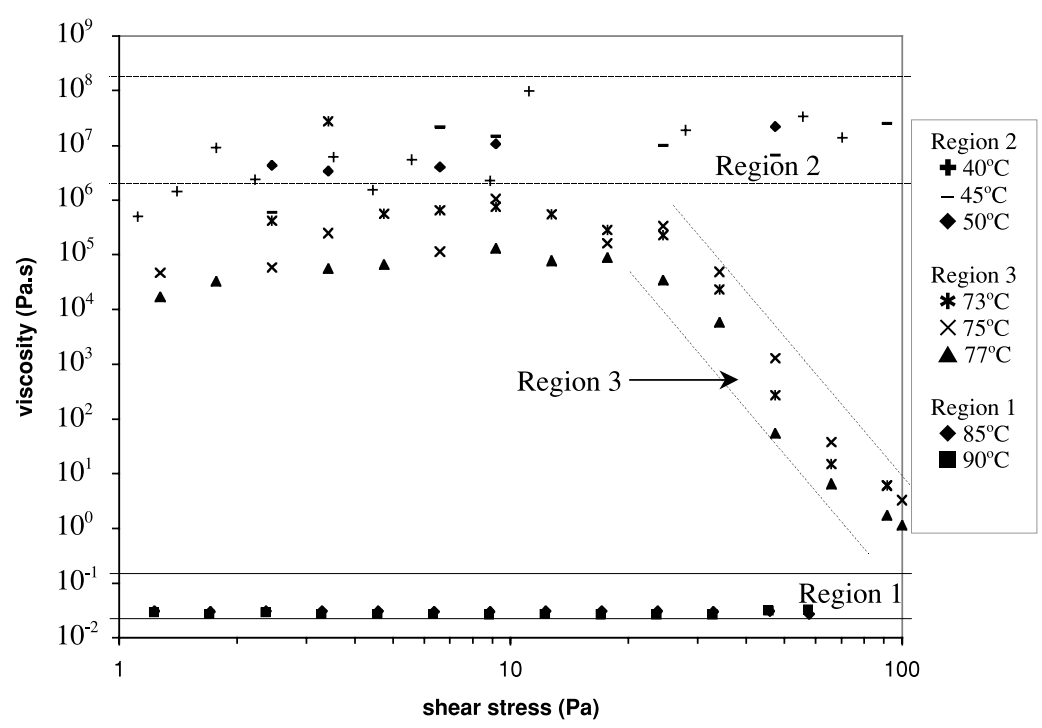

Figure 5. Plot of viscosity of the 75:25 mixture of M1595 and Lycojet waxes as a function of shear rate for different temperatures as measured on a constant stress rheometer.

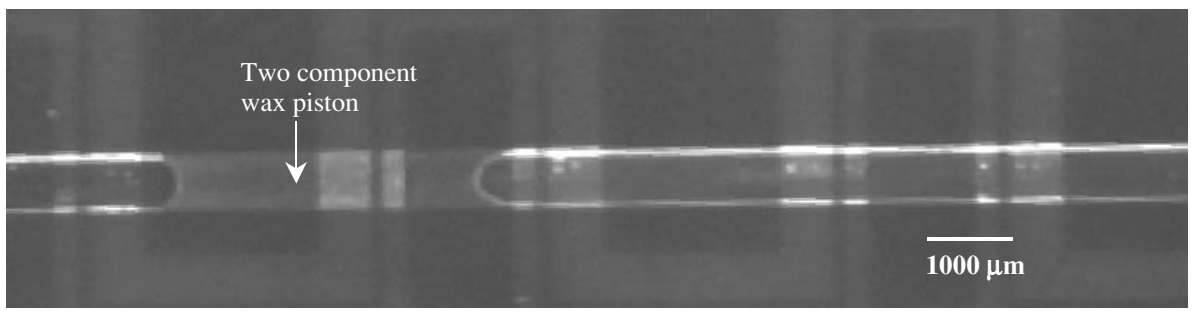

(a)

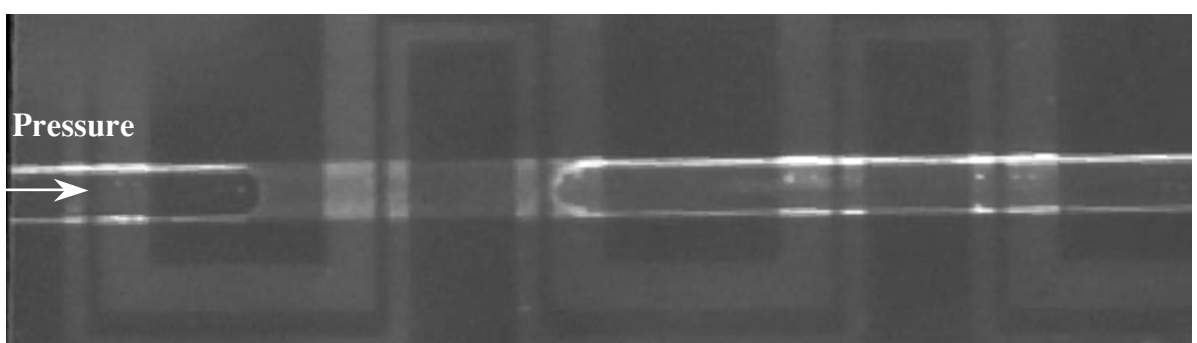

(b)

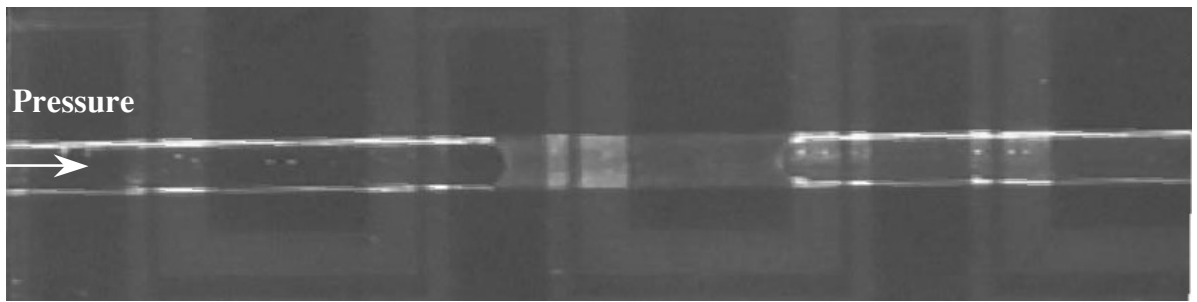

(c)

Figure 6. Pictures of the motion of a stable phase-change piston. (a) The initial plug. Parts $(b)$ and $(c)$ the slip-like motion of the piston in the microchannel. No coating of the microchannel was observed. The piston was about $3 \mathrm{~mm}$ long and a pressure of about $5 \mathrm{psi}$ $(\sim 34500 \mathrm{~Pa})$ was applied for the motion of the piston at a temperature of $75^{\circ} \mathrm{C}$.

A knowledge of this yield stress as a function of temperature is important for the design of such systems especially those involving multiple pistons.

\section{Gradient-less self-actuation}

As mentioned before, since most waxes exhibit a large decrease in viscosity as their temperature is raised, extremely localized heating is required so that both pistons do not yield at the same time. Alternatively, by using a piston made of thermoreversible material, gradient-less self-contained actuation can be obtained by global heating and cooling. Thermoreversible materials exhibit a step increase in viscosity as the temperature is raised beyond their phase transition temperature. Figure 8 shows the viscosity of a $12 \mathrm{wt} \%$ 


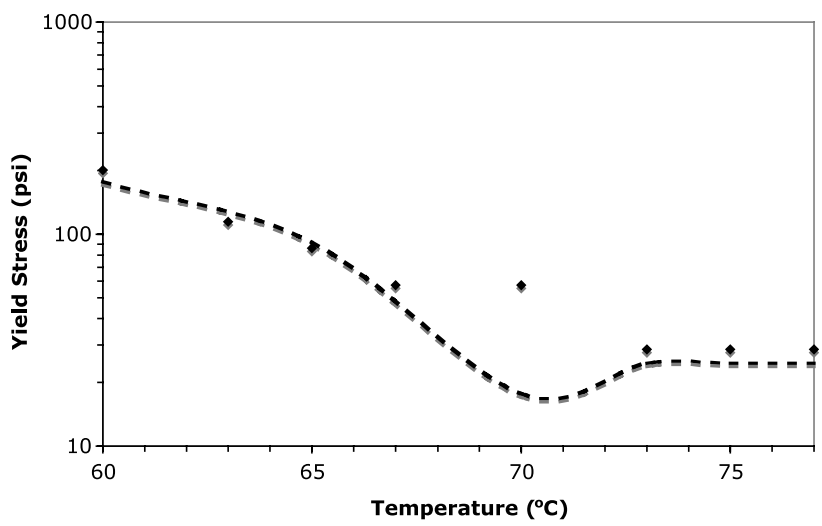

Figure 7. Plot of the yield stress as a function of temperature for a 75:25 mixture of M1595 and Lycojet waxes. The measurements were made in a silicon-glass microchannel $(50 \mu \mathrm{m}$ deep $\times 500 \mu \mathrm{m}$ wide). The dotted line represents the yield values measured by the rheometer.

solution of Pluronic ${ }^{\circledR}$ F127 in deionized water as a function of temperature. This unique thermal response characteristic of thermoreversible materials was exploited to eliminate the need for localized heating.

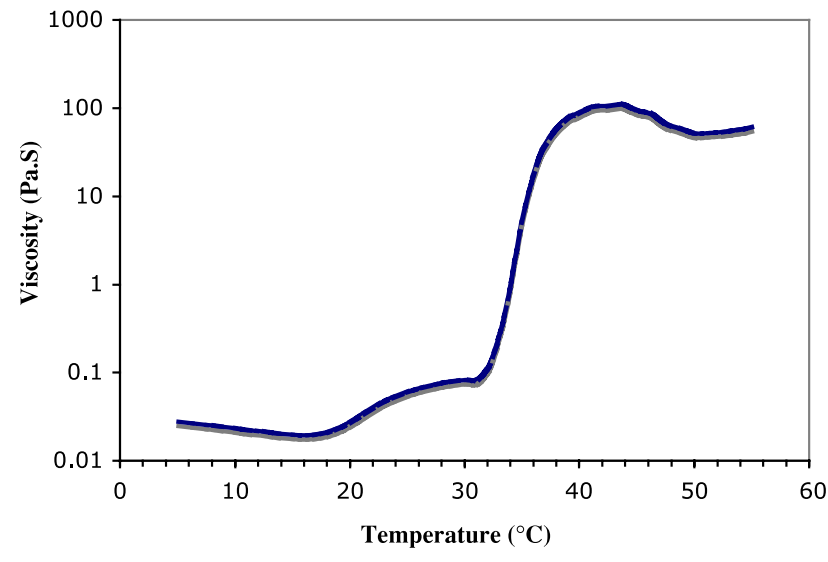

Figure 8. Plot of viscosity as a function of temperature for Pluronic ${ }^{\circledR}$ F-127 $(\sim 12 \mathrm{wt} \%)$. The measurements were made on a constant stress rheometer using the oscillatory mode at a frequency of $1 \mathrm{~Hz}$.

Figure 9 shows the actuation mechanism using two pistons where the second piston is made of a thermoreversible material. The wax piston is chosen such that it has a melting temperature $\left(T_{\mathrm{w}}\right)$ that is higher than the phase transition

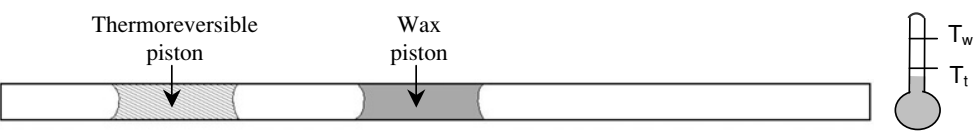

(a)

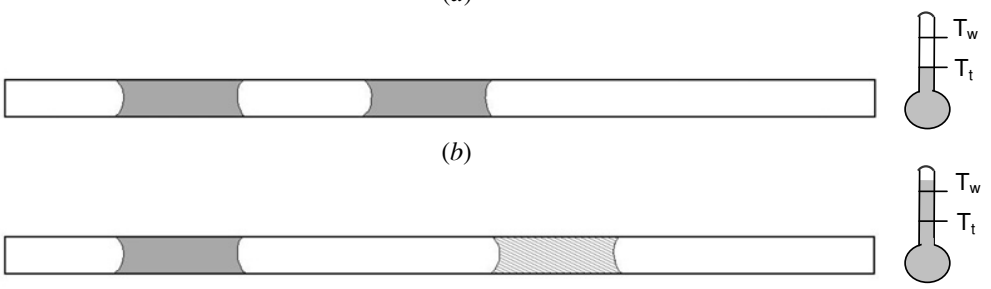

(c)

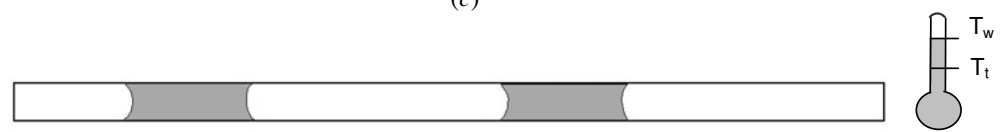

$(d)$

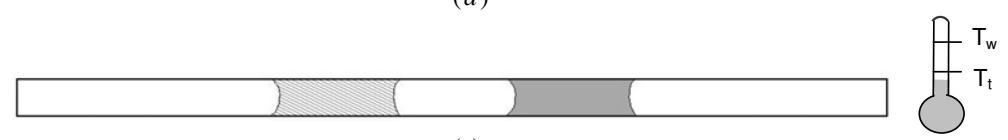

$(e)$

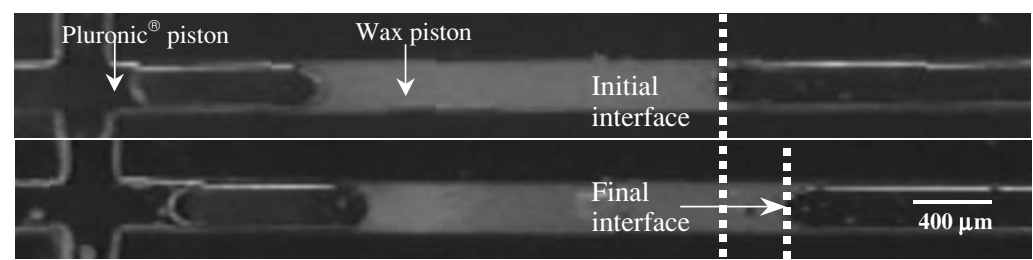

$(f)$

Figure 9. Operation schematics and pictures of an inchworm thermopneumatic pump using a wax piston and a thermoreversible piston. (a) At a temperature below $T_{\mathrm{t}}$ the wax piston is solid while the thermoreversible piston is in the liquid state. (b) As the temperature is raised to $T_{\mathrm{t}}$ the thermoreversible piston solidifies. $(c)$ When the temperature is raised further (beyond $T_{\mathrm{w}}$ ) the wax piston melts and the excess pressure created in the enclosed pocket propels the piston forward. (d) After this the temperature is lowered and as it drops below $T_{\mathrm{w}}$ the wax piston solidifies. (e) Lowering the temperature below $T_{\mathrm{t}}$ liquefies the thermoreversible piston that is pulled forward as the enclosed air contracts. $(f)$ Initial and final positions of the pistons for an inchworm thermopneumatic pump using a polyester wax piston and a Pluronic ${ }^{\circledR}$ piston in a $200 \mu \mathrm{m}$ wide $\times 50 \mu \mathrm{m}$ deep microchannel. 
temperature of thermoreversible material $\left(T_{\mathrm{t}}\right)$. At temperature below $T_{\mathrm{t}}$ the wax piston is solid while the thermoreversible is liquid. As the temperature is raised globally beyond $T_{\mathrm{w}}$ the thermoreversible piston first solidifies at about $T_{\mathrm{t}}$ and then the wax piston melts at $T_{\mathrm{w}}$. The excess pressure generated in the enclosed air pocket due to the increase in temperature propels the wax piston forward. As the temperature is reduced back below $T_{\mathrm{t}}$ the wax piston first solidifies, and then the thermoreversible piston liquefies and is pulled forward as the enclosed air contracts.

Figure $9(f)$ shows the implementation of this scheme using a thermoreversible piston of approximately $12 \mathrm{wt} \%$ Pluronic ${ }^{\circledR}$ F127 $\left(T_{\mathrm{t}} \sim 35{ }^{\circ} \mathrm{C}\right)$ and a wax piston that melts at about $37^{\circ} \mathrm{C}$. The microchannel was heated to about $40{ }^{\circ} \mathrm{C}$ on a hotplate to melt the wax piston and propel it forward. The Pluronic ${ }^{\circledR}$ F127 was liquefied by placing the microdevice in a refrigerator to lower the temperature of the device to about $5{ }^{\circ} \mathrm{C}$. As seen from the figure, after two cycles of heating and cooling a forward displacement of $\sim 200 \mu \mathrm{m}$ was obtained. Thus this scheme can be used for unidirectional self-contained actuation by global heating and cooling of the microdevice.

One problem associated with using thermoreversible material is the degradation of the matrix due to the evaporation of the solvent at elevated temperatures. The evaporation of the solvent causes a shift in the phase-change temperature [13] and excessive loss of solvent leaves behind a white powder. This phenomenon is exaggerated in the microchannels due the high surface to volume ratio. The problem was solved in our work by introducing a plug of water behind the Pluronic ${ }^{\circledR}$ piston to arrest the evaporation of water from the matrix. The introduction of a plug of solvent or any other non-volatile liquid does not effect the self-contained actuation. This scheme for arresting the evaporation of the solvent can also be implemented in other microfluidic applications involving thermoreversible materials.

\section{Conclusions}

A self-contained actuation mechanism using two phasechange pistons and a moving thermopneumatic chamber has been demonstrated. This process eliminates the requirement for large air chambers and high temperatures typically associated with on-chip thermopneumatic pumping. The self-contained actuation mechanism can be coupled with advanced thermal isolation and microchannel designs to perform microfluidic manipulations. A mechanism for stabilizing the wax piston such that it does not coat the wall has also been presented. This mechanism that introduces a 'slip' layer between the piston and wall can be employed for a variety of microfluidics operations where high pressure requirements due to the reduced size are a limiting factor. Actuation principles presented in this work can be modified and characterized to realize valving and pumping operations on microfluidic-integrated devices.

\section{Acknowledgments}

The authors would like to thank Professor Ronald G Larson for discussions on clarifying the molten piston motion. The authors would also like to thank Siva Vanapalli and Anshuman Roy for useful discussions. The authors would like to acknowledge the National Institute of Health for funding this project under grant P01-HG001984.

\section{References}

[1] Shoji S and Esashi M 1994 Microflow devices and systems J. Micromech. Microeng. 4 157-71

[2] Unger M A, Chou H P, Thorsen T, Scherer A and Quake S R 2000 Monolithic microfabricated valves and pumps by multilayer soft lithography Science 288 113-6

[3] Grover W H, Skelley A M, Liu C N, Lagally E T and Mathies R A 2003 Monolithic membrane valves and diaphragm pumps for practical large-scale integration into glass microfluidic devices Sensors Actuators 89 315-23

[4] Gu W, Zhu X Y, Futai N, Cho B S and Takayama S 2004 Computerized microfluidic cell culture using elastomeric channels and Braille displays Proc. Natl Acad. Sci. USA 101 15861-6

[5] Weibel D B, Kruithof M, Potenta S, Sia S K, Lee A and Whitesides G M 2005 Torque-actuated valves for microfluidics Anal. Chem. 77 4726-33

[6] Hasselbrink E F, Shepodd T J and Rehm J E 2002 High-pressure microfluidic control in lab-on-a-chip devices using mobile polymer monoliths Anal. Chem. 74 4913-8

[7] Eddington D T and Beebe D J 2004 Flow control with hydrogels Adv. Drug Deliv. Rev. 56 199-210

[8] Yu C, Mutlu S, Selvaganapathy P, Mastrangelo C H, Svec F and Frechett J M J 2003 Flow control valves for analytical microfluidic chips without mechanical parts based on thermally responsive monolithic polymers Anal. Chem. 75 1958-61

[9] Hartshorne H, Backhouse C J and Lee W E 2004 Ferrofluid-based microchip pump and valve Sensors Actuators B 99 592-600

[10] Yamahata C, Chastellain M, Parashar V K, Petri A, Hofmann H and Gijs M A M 2005 Plastic micropump with ferrofluidic actuation J. Microelectromech. Syst. 14 96-102

[11] Yoshida K, Kikuchi M, Park J H and Yokota S 2002 Fabrication of micro electro-rheological valves (ER valves) by micromachining and experiments Sensors Actuators A 95 227-33

[12] Gui L and Liu J 2004 Ice valve for a mini/micro flow channel J. Micromech. Microeng. 14 242-6

[13] Stoeber B, Yang Z H, Liepmann D and Muller S J 2005 Flow control in microdevices using thermally responsive triblock copolymers J. Microelectromech. Syst. 14 207-13

[14] Liu Y J, Rauch C B, Stevens R L, Lenigk R, Yang J N, Rhine D B and Grodzinski P 2002 DNA amplification and hybridization assays in integrated plastic monolithic devices Anal. Chem. 74 3063-70

[15] Pal R et al 2005 An integrated microfluidic device for influenza and other genetic analyses $L a b$ Chip 5 1024-32

[16] Liu R H, Yang J N, Lenigk R, Bonanno J and Grodzinski P 2004 Self contained, fully integrated biochip for sample preparation, polymerase chain reaction amplification, and DNA microarray detection Anal. Chem. 76 1824-31

[17] Pal R, Yang M, Johnson B N, Burke D T and Burns M A 2004 Phase change microvalve for integrated devices Anal. Chem. 76 3740-8

[18] Liu R H, Bonanno J, Yang J N, Lenigk R and Grodzinski P 2004 Sensors Actuators B 98 328-36 
[19] Pal R, Sung K E and Burns M A 2006 Microstencils for patterning non-traditional materials unpublished

[20] Yang M, Pal R and Burns M A 2005 Cost-effective thermal isolation techniques for use on microfabricated DNA amplification and analysis device J. Micromech. Microeng. 15 221-31
[21] Sammarco T S and Burns M A 1999 Thermocapillary pumping of discrete drops in microfabricated analysis device AIChE J. 45 350-66

[22] Polinski A J, Oehler P R and Stokes V R 1995 Isothermal gas-assisted displacement of viscoplastic fluids in tubes Polym. Eng. Sci. 35 877-92 